\title{
The comparison of fluticasone propionate/formoterol with fluticasone propionate/salmeterol for paediatric asthma: a meta-analysis of randomized controlled trials
}

\author{
Xiaofen Jin ${ }^{1}$, Lianfang $\mathrm{Wu}^{2}$, Ji Wang ${ }^{3}$, Malong Feng ${ }^{3}$ \\ 'Department of Pediatrics, Fenghua District People's Hospital of Ningbo, Zhejiang, China \\ 2Department of Pediatrics, Ningbo Yinzhou Second Hospital, Zhejiang, China \\ ${ }^{3}$ Department of Respiration, Fenghua District People's Hospital of Ningbo, Zhejiang, China
}

Adv Dermatol Allergol 2021; XXXVIII (3): 377-383

DOI: https://doi.org/10.5114/ada.2020.92519

\begin{abstract}
Introduction: The comparison of fluticasone propionate/formoterol (FP/FORM) with fluticasone propionate/salmeterol (FP/SAL) for paediatric asthma remains controversial.

Aim: We conduct a systematic review and meta-analysis to explore the efficacy and safety of FP/FORM versus FP/SAL for paediatric asthma.

Material and methods: We have searched PubMed, Embase, Web of science, EBSCO, and Cochrane library databases through August 2019 for randomized controlled trials (RCTs) assessing the effect of FP/FORM versus FP/SAL for paediatric asthma. This meta-analysis is performed using the random-effects model.

Results: Three RCTs are included in the meta-analysis. Overall for paediatric asthma, FP/FORM and FP/SAL demonstrate a comparable influence on $\mathrm{FEV}_{1}$ (Std. MD $=-0.01 ; 95 \% \mathrm{Cl}:-0.04$ to $\left.0.03 ; p=0.62\right)$, FVC (Std. MD = 0; $95 \% \mathrm{Cl}:-0.07$ to $0.06 ; p=0.87$ ), FEF25 (Std. MD = $-1.69 ; 95 \% \mathrm{Cl}:-6.69$ to 3.31; $p=0.51$ ), FEF50 (Std. MD = 0.10; $95 \% \mathrm{Cl}:-0.12$ to $0.33 ; p=0.37$ ), FEF75 (Std. $\mathrm{MD}=0.01 ; 95 \% \mathrm{Cl}:-0.21$ to $0.24 ; p=0.91$ ), asthma symptom scores (Std. MD $=-0.03 ; 95 \% \mathrm{Cl}:-0.11$ to $0.04 ; p=0.43$ ), sleep disturbance scores (Std. $\mathrm{MD}=0.03 ; 95 \% \mathrm{Cl}:-0.19$ to 0.24 ; $p=0.81)$ and adverse events ( $\mathrm{RR}=1.07 ; 95 \% \mathrm{Cl}: 0.83$ to $1.38 ; p=0.61)$.
\end{abstract}

Conclusions: FP/FORM and FP/SAL show a comparable efficacy for paediatric asthma.

Key words: fluticasone propionate/formoterol, propionate/salmeterol, paediatric asthma, randomized controlled trials, meta-analysis.

\section{Introduction}

Asthma has become one of the most common chronic medical conditions and it is estimated that around 300 million people globally suffer from asthma. Among them, the number in children is gradually increasing [1-3]. Underlying inflammation is crucial for the pathophysiology of asthma, and is associated with bronchial hyper-responsiveness, airway obstruction, and respiratory symptoms $[4,5]$. Chronic inflammation and subsequent structural changes lead to persistent symptoms and reduced lung function $[6,7]$. Asthma (GINA) guidelines recommend inhaled corticosteroid and long-acting $\beta 2$-agonist (ICS/LABA) combinations as the Step 3 controller option in children aged 6-11 years [8]. Single-inhaler combination ICS/LABA therapy is reported to increase treatment adherence and may improve treatment outcomes better than free combinations of ICS and LABA $[9,10]$.

Fluticasone propionate is an effective ICS, and has sustained anti-inflammatory effects. Formoterol fumarate is the most rapid-acting LABA, and shows the speed of onset comparable to the short-acting $\beta 2$-agonist, salbutamol. Flutiform ${ }^{\circledR}$, fluticasone propionate and formoterol fumarate (FP/FORM) combination therapy has revealed the efficacy for adults and/or adolescents with mild to severe asthma, and has been approved for use in over 30 countries [11-15]. An open-label, randomized, controlled, phase III trial involving 211 patients compares FP/FORM with FP/SAL in paediatric asthmatic patients, and the results show that they have comparable lung function improvement and asthma control [16].

Address for correspondence: Malong Feng, Department of Pediatrics, Fenghua District People's Hospital of Ningbo, 6 Seven Branch Panxi Road, Jiangbei District, Fenghua, Zhejiang 300061, China, phone: +86 02389342584 , fax: +86 02389342584, e-mail: thursday13@163.com

Received: 5.10.2019, accepted: 28.10.2019. 
Recently, several studies have reported FP/FORM versus FP/SAL for paediatric asthma have been published, but their efficacy has not been well established [16-18].

\section{Aim}

With accumulating evidence, we therefore perform a systematic review and meta-analysis of randomized controlled trials (RCTs) to compare their efficacy for paediatric asthma patients.

\section{Material and methods}

Ethical approval and patient consent are not required because this is a systematic review and meta-analysis of previously published studies. The systematic review and meta-analysis is conducted and reported in adherence to PRISMA (Preferred Reporting Items for Systematic Reviews and Meta-Analyses) [19].

\section{Search strategy and study selection}

Two investigators have independently searched the following databases (inception to August 2019): PubMed, Embase, Web of science, EBSCO, and Cochrane library databases. The electronic search strategy is conducted using the following key words: fluticasone propionate, and formoterol, and salmeterol, and asthma, and paediatric or children. We also check the reference lists of the screened full-text studies to identify other potentially eligible trials.

The inclusion criteria are as follows: (i) population: patients are diagnosed with paediatric asthma; (ii) intervention treatments are FP/FORM versus FP/SAL; (iii) study design is RCT.

\section{Data extraction and outcome measures}

We have extracted the following information: author, number of patients, age, female, forced expiratory volume in $1 \mathrm{~s}\left(\mathrm{FEV}_{1}\right)$, inhaled corticosteroid use and detailed methods in each group etc. Data have been extracted independently by two investigators, and discrepancies are resolved by consensus. We also contact the corresponding author to obtain the data when necessary.

The primary outcome is FEV . Secondary outcomes include forced vital capacity (FVC), forced expiratory flow at 25\% (FEF25), FEF50, FEF75, asthma symptom scores, sleep disturbance scores, and adverse events.

\section{Quality assessment in individual studies}

Methodological quality of the included studies is independently evaluated using the modified Jadad scale [20]. There are 3 items for Jadad scale: randomization (0-2 points), blinding ( $0-2$ points), dropouts and withdrawals (0-1 points). The score of Jadad scale varies from 0 to 5 points. An article with Jadad score of $\leq 2$ is considered to be of low quality. If the Jadad score is $\geq 3$, the study is thought to be of high quality [21].

\section{Statistical analysis}

We estimate the standard mean difference (Std. MD) with 95\% confidence interval (CI) for continuous outcomes (FEV ${ }_{1}$, FVC, FEF25, FEF50, FEF75, asthma symptom scores, and sleep disturbance scores) and risk ratio (RR) with $95 \% \mathrm{Cl}$ for dichotomous outcomes (adverse events). A random-effects model is used regardless of heterogeneity [22]. Heterogeneity is reported using the $P$ statistic, and $P>50 \%$ indicates significant heterogeneity $[22,23]$. Whenever significant heterogeneity is present, we search for potential sources of heterogeneity via omitting one study in turn for the meta-analysis or performing subgroup analysis. All statistical analyses are performed using Review Manager Version 5.3 (The Cochrane Collaboration, Software Update, Oxford, UK).

\section{Results}

\section{Literature search, study characteristics and quality} assessment

A detailed flowchart of the search and selection results is shown in Figure 1. Three hundred and fifty-five potentially relevant articles are identified initially. Finally, two full articles and one abstract that meet our inclusion criteria are included in the meta-analysis [16-18].

The baseline characteristics of the three eligible RCTs in the meta-analysis are summarized in Table 1 . The three studies are published between 2010 and 2018, and sample sizes range from 202 to 335 with a total of 748 . Two studies report inhaled fluticasone/formoterol (100/10 $\mu \mathrm{g}$ bid) versus fluticasone/salmeterol (100/50 $\mu \mathrm{g}$ bid) for 12 weeks $[16,17]$, the remaining abstract reports fluticasone/formoterol (100 or 250/10 $\mu \mathrm{g}$ bid) versus fluticasone/salmeterol (100 or 250/50 ug bid) for 12 weeks [18].

Among the three studies included here, two studies report FEV , FVC, FEF25, FEF50, FEF75, asthma symptom scores and sleep disturbance scores $[16,17]$, and three studies report adverse events [16-18]. Jadad scores of the three included studies vary from 3 to 5 , and all three studies are considered to be high-quality ones according to quality assessment.

\section{Primary outcome: $\mathrm{FEV}_{1}$}

The outcome data are analysed with the randomeffects model, and compared to inhaled FP/SAL for paediatric asthma, FP/FORM has a similar impact on FEV (Std. $M D=-0.01 ; 95 \% \mathrm{Cl}:-0.04$ to $0.03 ; p=0.62$ ) with no heterogeneity among the studies $\left(R^{2}=0 \%\right.$, heterogeneity $p=0.40$ ) (Figure 2).

\section{Sensitivity analysis}

No heterogeneity is observed for the primary outcomes, and thus we do not perform the sensitivity analy- 


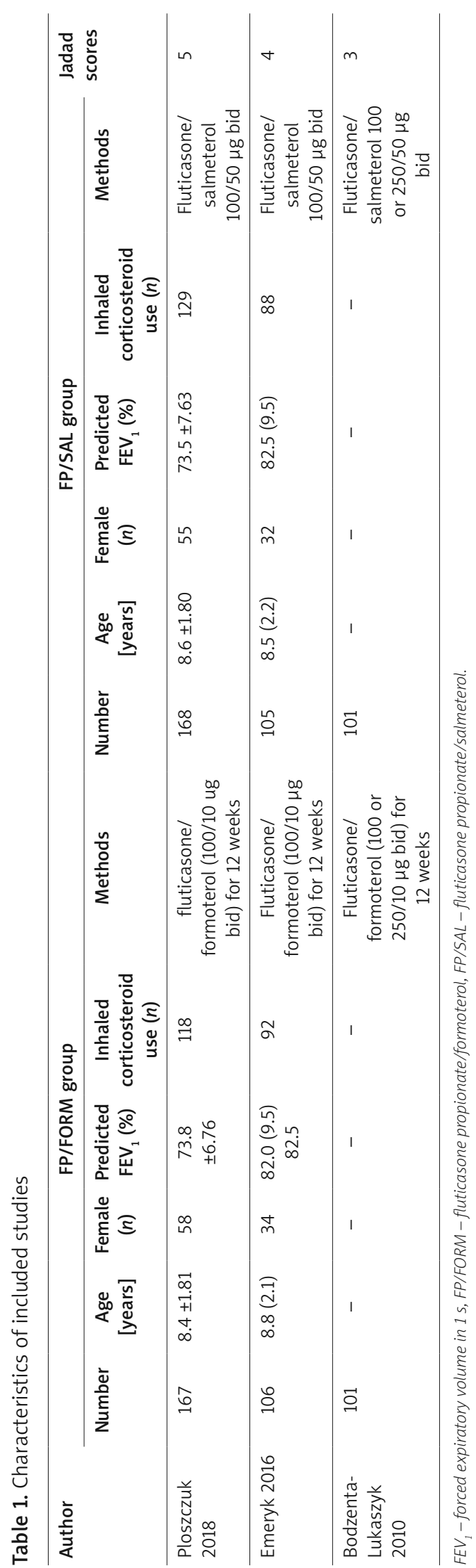

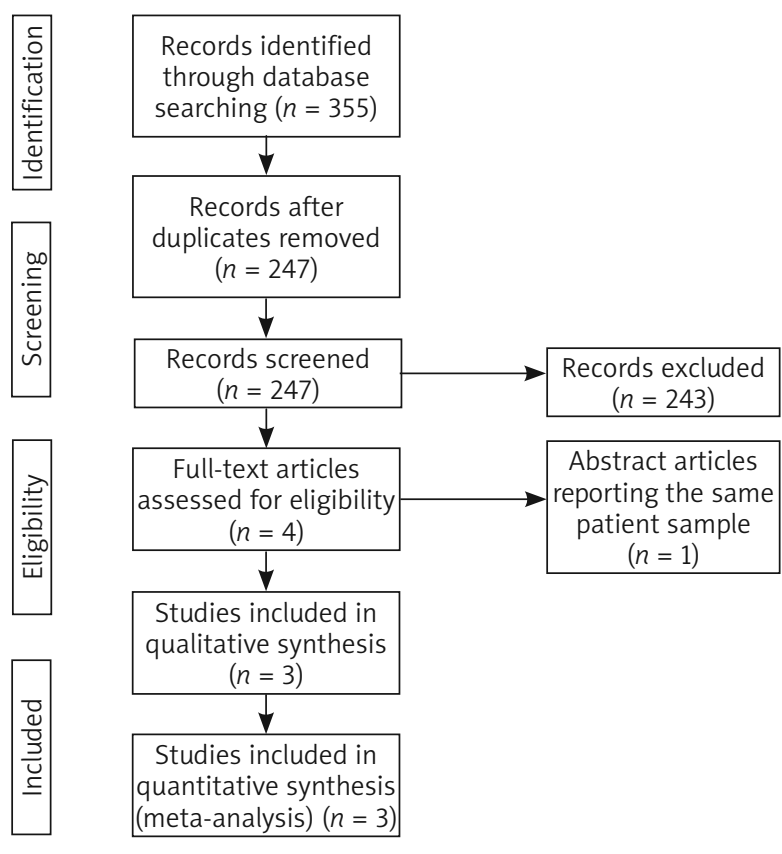

Figure 1. Flow diagram of study searching and selection process

sis by omitting one study in order to detect the heterogeneity.

\section{Secondary outcomes}

In comparison with inhaled FP/SAL for paediatric asthma, FP/FORM demonstrates a comparable effect on FVC (Std. MD = 0; 95\% Cl: -0.07 to 0.06; $p=0.87$; Figure 3), FEF25 (Std. MD = -1.69; 95\% Cl: -6.69 to 3.31; $p=0.51$; Figure 4), FEF50 (Std. MD $=0.10 ; 95 \% \mathrm{Cl}$ : -0.12 to 0.33; $p=0.37$; Figure 5), FEF75 (Std. MD =0.01; $95 \% \mathrm{Cl}:-0.21$ to $0.24 ; p=0.91$; Figure 6 ), asthma symptom scores (Std. MD $=-0.03 ; 95 \% \mathrm{Cl}$ : -0.11 to $0.04 ; p=0.43$; Figure 7), sleep disturbance scores (Std. MD =0.03; 95\% Cl: -0.19 to $0.24 ; p=0.81$; Figure 8 ) and adverse events ( $R R=1.07 ; 95 \% \mathrm{Cl}$ : 0.83 to $1.38 ; p=0.61$; Figure 9 ).

\section{Discussion}

Low-dose inhaled corticosteroids (ICSS) are highly effective to decrease the symptoms and the risk of asthma exacerbations, and initiation of ICS treatment or in combination with short-acting $\beta 2$-agonist (SABA) early in the disease course is recommended in patients with the risk of exacerbations for inhibiting a long-term decline in lung function [1, 24-27]. ICS and the long-acting $\beta 2$ agonist (LABA) combination treatment is recommended for children with uncontrolled asthma after low-dose ICS treatment based on the GINA guidelines [8].

Addition of LABA to low-dose ICS may result in best response than increasing the dose of ICS [28]. Previous 


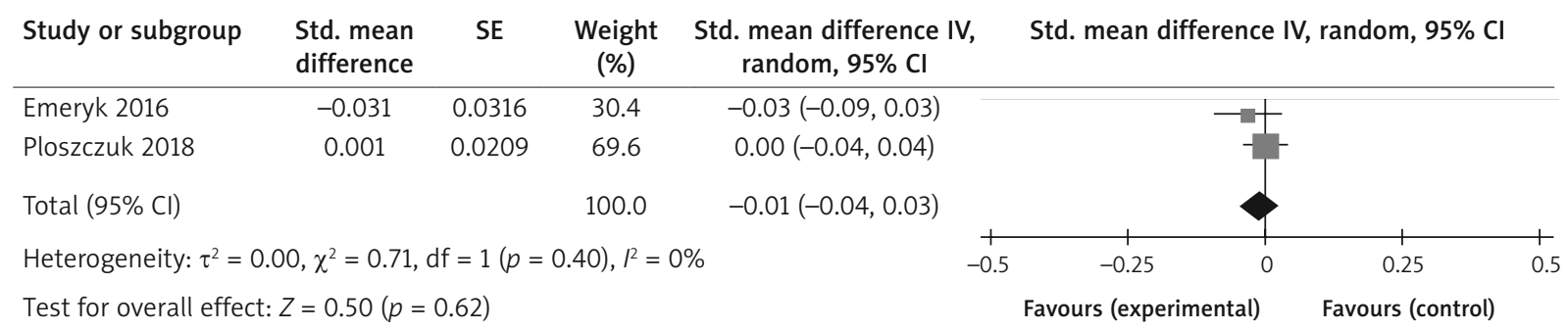

Figure 2. Forest plot for the meta-analysis of FEV

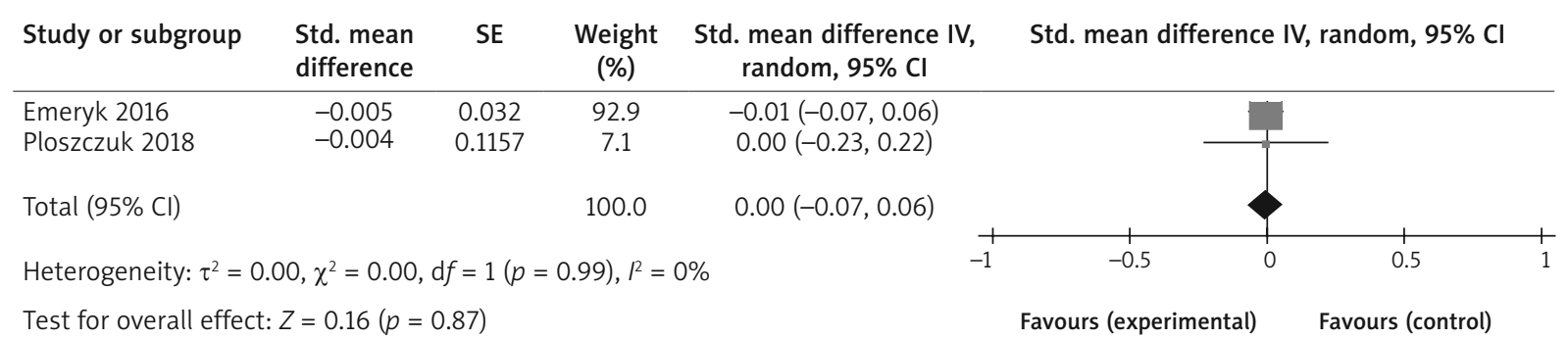

Figure 3. Forest plot for the meta-analysis of FVC (l)

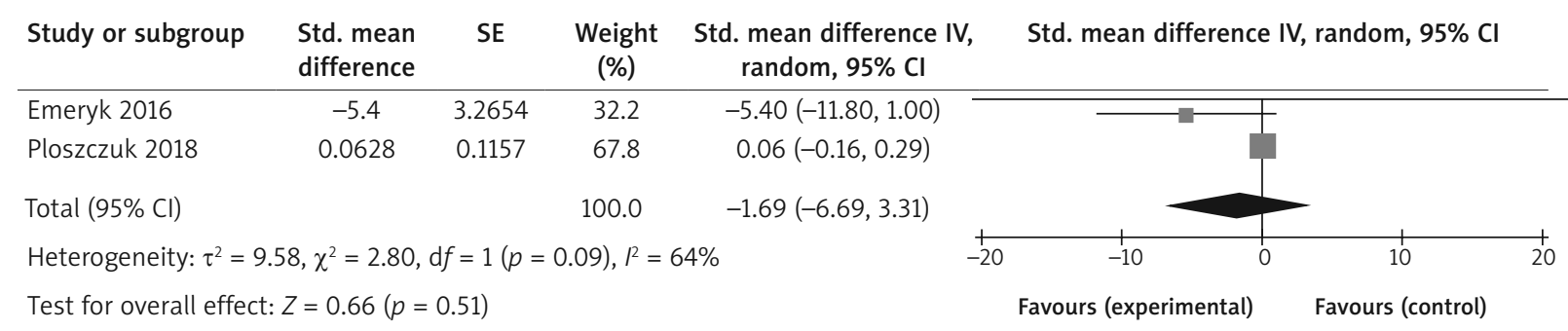

Figure 4. Forest plot for the meta-analysis of FEF25 (I/s)

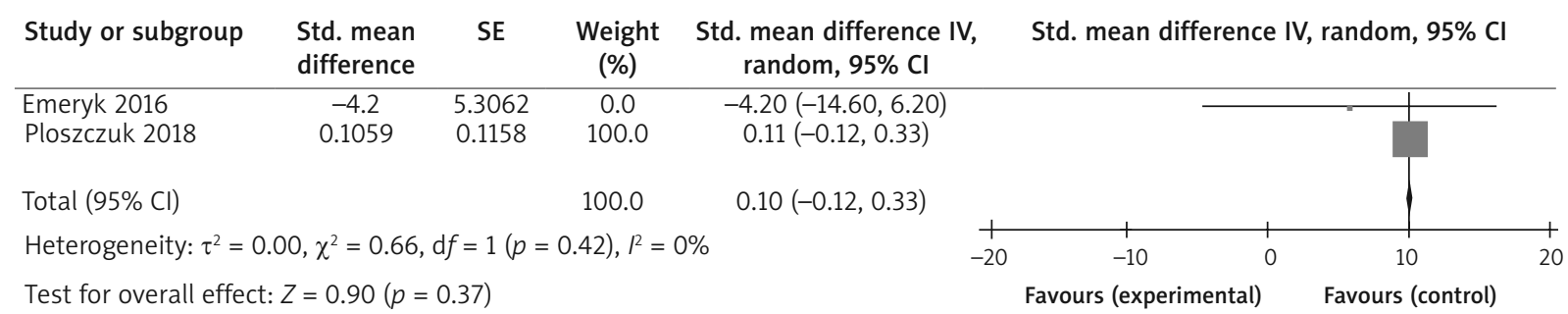

Figure 5. Forest plot for the meta-analysis of FEF50 (l/s)

studies demonstrate the non-inferiority of FP/FORM compared with other ICS/LABA combinations in adolescents and adults with asthma [11, 29, 30]. Two ICS/LABA combination therapies are currently used in children, and they include fluticasone/salmeterol as both a dry powder inhaler (DPI) and pressurized metered-dose inhaler (pMDI) and budesonide/formoterol DPI [17].

Our meta-analysis suggests that FP/FORM and FP/ SAL have comparable lung function, asthma symptom scores and sleep disturbance scores in paediatric asthma. There is a modest increase in mean FEV of $105 \mathrm{ml}$ during the extension phase, predicted $\mathrm{FEV}_{1}$ remains very stable between day 84 and day 252, suggesting that the increase in $\mathrm{FEV}_{1}$ may be mainly caused by the growth of children during the 24-week extension phase [16]. There are a low number of patients who experienced asthma exacerbations during either FP/FORM or FP/SAL treatment (4 (3.8\%) FP/FORM patients and 3 (2.9\%) FP/SAL 


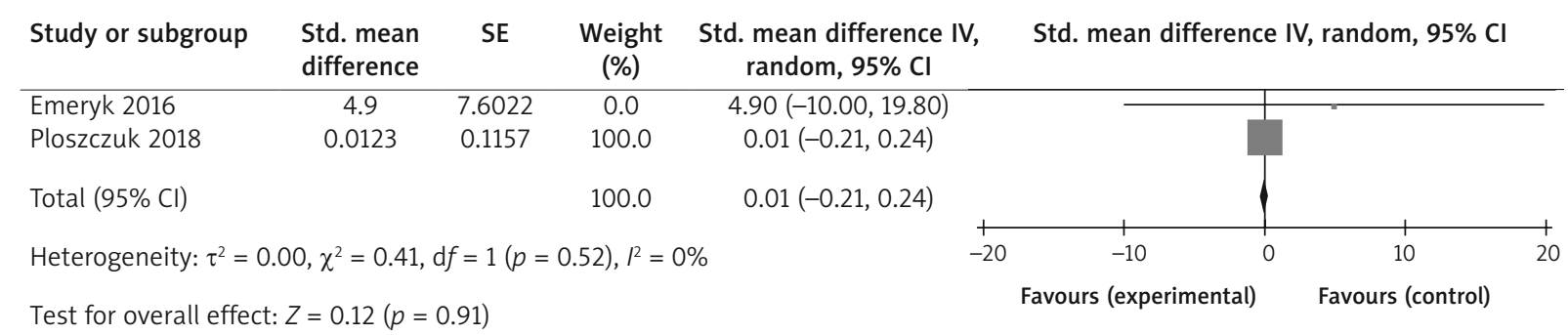

Figure 6. Forest plot for the meta-analysis of FEF75 (l/s)

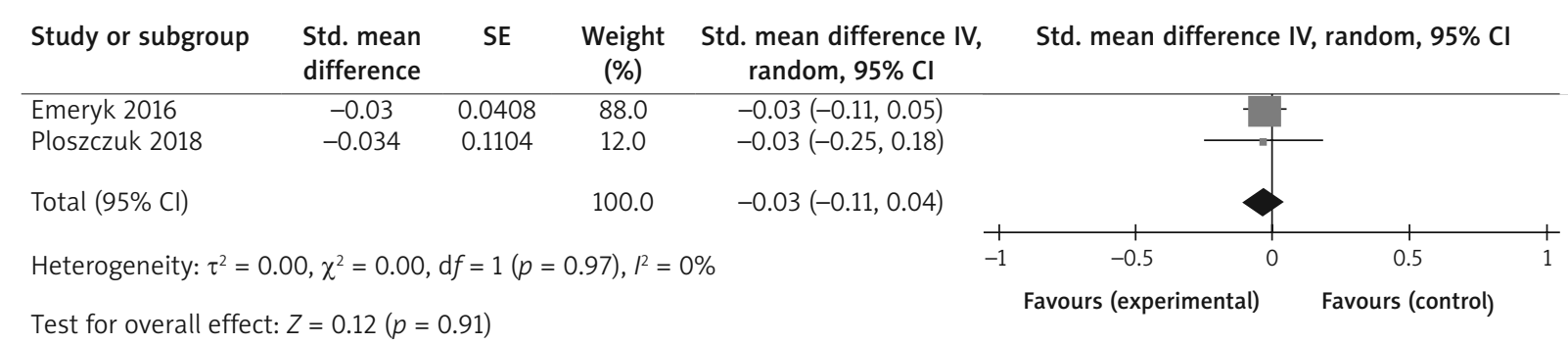

Figure 7. Forest plot for the meta-analysis of asthma symptom scores

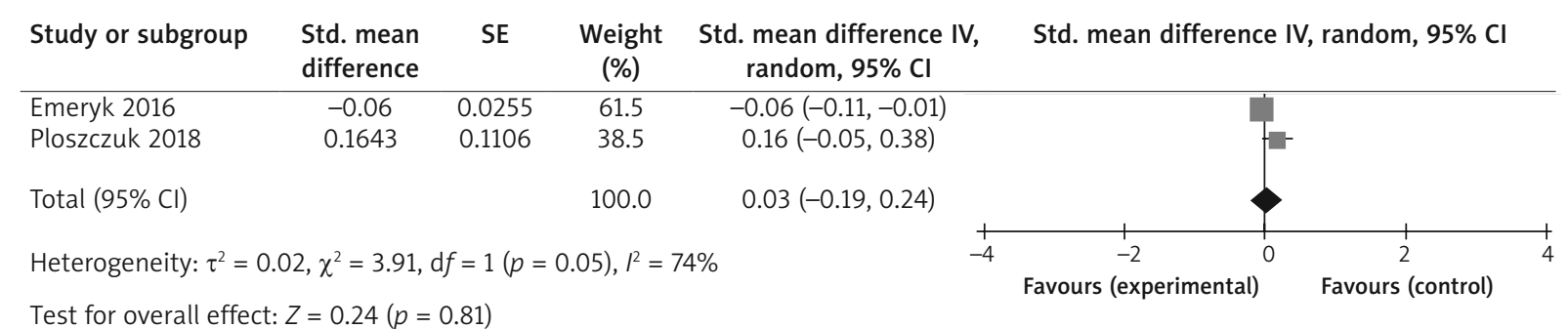

Figure 8. Forest plot for the meta-analysis of sleep disturbance scores

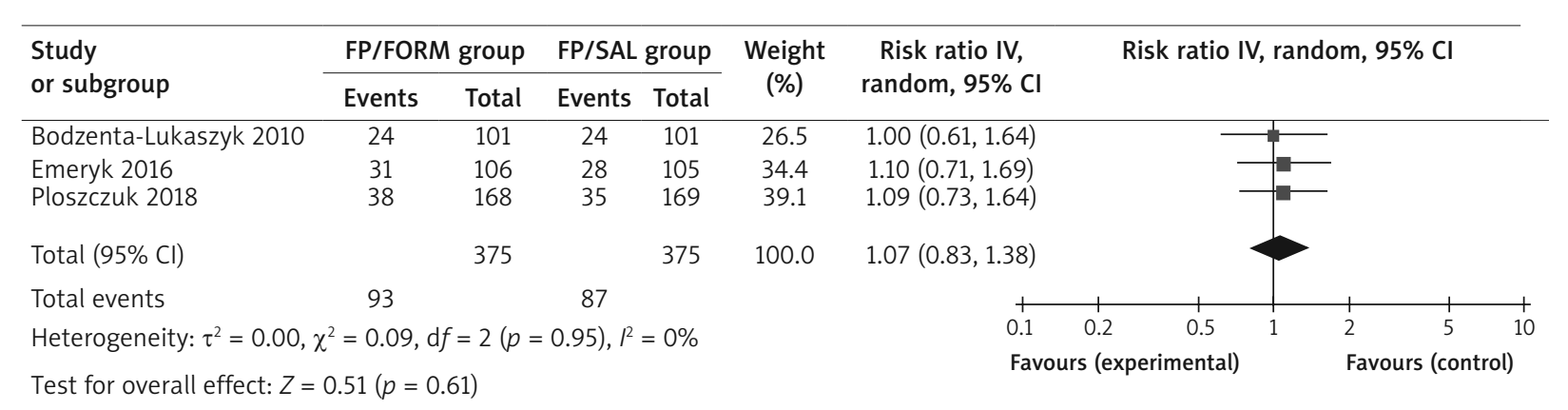

Figure 9. Forest plot for the meta-analysis of adverse events

patients), and may show a lower incidence of any exacerbation type compared with FP monotherapy [30].

FP/FORM has a favourable safety and tolerability profile throughout the 24-week extension phase in paediatric patients, and shows no effect on normal growth $[16,31,32]$. There is a similar incidence of adverse events between FP/FORM and FP/SAL for paediatric asthma in this meta-analysis. In addition, 6-12 months of treatment with FP/FORM may be associated with a lower incidence of severe asthma exacerbations than single-inhaler FP/ SAL and budesonide/formoterol, which may be related to the favourable pharmacological/mechanistic characteristics of the constituent components: fluticasone and formoterol compared to other drugs [32]. This meta-analysis 
has several potential limitations. Firstly, our analysis is based on three RCTs, and more RCTs with a large sample size should be conducted to explore this issue. Next, different doses of drugs and time for drug use may have some influence on the pooling results. Finally, some unpublished and missing data may lead to some bias to the pooled effect.

\section{Conclusions}

FP/FORM and FP/SAL demonstrate similar lung function improvement and asthma control for paediatric asthma.

\section{Acknowledgments}

Xiaofen Jin and Lianfang Wu contribute equally.

\section{Conflict of interest}

The authors declare no conflict of interest.

\section{References}

1. (GINA) GIfA. Global strategy for asthma management and prevention. Available from: wwwginasthmaorg. Accessed: 25 March 2015.

2. Wenzel SE, Jayawardena S, Graham NM, et al. Severe asthma and asthma-chronic obstructive pulmonary disease syndrome - Authors' reply. Lancet 2016; 388: 2742.

3. Pavord ID, Beasley R, Agusti A, et al. After asthma: redefining airways diseases. Lancet 2018; 391: 350-400.

4. Murphy DM, O'Byrne PM. Recent advances in the pathophysiology of asthma. Chest 2010; 137: 1417-26.

5. Fahy JV. Type 2 inflammation in asthma - present in most, absent in many. Nat Rev Immunol 2015; 15: 57-65.

6. Reddel HK, Taylor DR, Bateman ED, et al. An official American Thoracic Society/European Respiratory Society statement: asthma control and exacerbations: standardizing endpoints for clinical asthma trials and clinical practice. Am J Respir Crit Care Med 2009; 180: 59-99.

7. Ray A, Kolls JK. Neutrophilic inflammation in asthma and association with disease severity. Trends Immunol 2017; 38: 942-54.

8. Asthma. GIf. GINA Global Strategy for Asthma Management and Prevention. 2016, http://ginasthma.org/.

9. Chroinin MN, Lasserson TJ, Greenstone I, Ducharme FM. Addition of long-acting beta-agonists to inhaled corticosteroids for chronic asthma in children. Cochrane Database Syst Rev 2009; 3: CD007949.

10. Murphy KR, Bender BG. Treatment of moderate to severe asthma: patient perspectives on combination inhaler therapy and implications for adherence. J Asthma Allergy 2009; 2: 63-72.

11. Bodzenta-Lukaszyk A, Dymek A, McAulay K, Mansikka H. Fluticasone/formoterol combination therapy is as effective as fluticasone/salmeterol in the treatment of asthma, but has a more rapid onset of action: an open-label, randomized study. BMC Pulm Med 2011; 11: 28.

12. Nathan RA, D'Urzo A, Blazhko V, Kaiser K. Safety and efficacy of fluticasone/formoterol combination therapy in adolescent and adult patients with mild-to-moderate asthma: a randomised controlled trial. BMC Pulm Med 2012; 12: 67.
13. Bodzenta-Lukaszyk A, Buhl R, Balint B, et al. Fluticasone/formoterol combination therapy versus budesonide/formoterol for the treatment of asthma: a randomized, controlled, noninferiority trial of efficacy and safety. J Asthma 2012; 49: 1060-70.

14. Corren J, Mansfield LE, Pertseva T, et al. Efficacy and safety of fluticasone/formoterol combination therapy in patients with moderate-to-severe asthma. Respir Med 2013; 107: 180-95.

15. Bodzenta-Lukaszyk A, van Noord J, Schröder-Babo W, et al. Efficacy and safety profile of fluticasone/formoterol combination therapy compared to its individual components administered concurrently in asthma: a randomised controlled trial. Curr Med Res Opin 2013; 29: 579-88.

16. Emeryk A, Klink R, Mclver T, Dalvi P. A 12-week open-label, randomized, controlled trial and 24-week extension to assess the efficacy and safety of fluticasone propionate/formoterol in children with asthma. Ther Adv Respir Dis 2016; 10: 324-37.

17. Ploszczuk A, Bosheva M, Spooner K, et al. Efficacy and safety of fluticasone propionate/formoterol fumarate in pediatric asthma patients: a randomized controlled trial. Ther Adv Respir Dis 2018; 12: 1753466618777924.

18. Bodzenta-Lukaszyk A, Dymek A, Mansikka H. P24 Fluticasone propionate/formoterol fumarate combination therapy has a more rapid onset of action than fluticasone propionate/ salmeterol xinafoate in the treatment of asthma: a randomised controlled trial. Thorax 2010; 65: A86-A.

19. Moher D, Liberati A, Tetzlaff J, Altman DG; PRISMA Group. Preferred reporting items for systematic reviews and metaanalyses: the PRISMA statement. J Clin Epidemiol 2009; 62: 1006-12.

20.Jadad AR, Moore RA, Carroll D, et al. Assessing the quality of reports of randomized clinical trials: Is blinding necessary? Control Clin Trials 1996; 17: 1-12.

21. Kjaergard LL, Villumsen J, Gluud C. Reported methodologic quality and discrepancies between large and small randomized trials in meta-analyses. Ann Intern Med 2001; 135: 982 9.

22.Zhao J, Huang W, Zhang S, et al. Efficacy of glutathione for patients with cystic fibrosis: a meta-analysis of randomized-controlled studies. Am J Rhinol Allergy 2019; 1945892419878315.

23. Higgins JP, Thompson SG. Quantifying heterogeneity in a meta-analysis. Stat Med 2002; 21: 1539-58.

24. Paggiaro P, Halpin DM, Buhl R, et al. The effect of tiotropium in symptomatic asthma despite low-to medium-dose inhaled corticosteroids: a randomized controlled trial. J Allergy Clin Immunol Pract 2016; 4: 104-13.e2.

25. Eltonsy S, Forget A, Beauchesne MF, Blais L. Risk of congenital malformations for asthmatic pregnant women using a long-acting 2-agonist and inhaled corticosteroid combination versus higher-dose inhaled corticosteroid monotherapy. J Allergy Clin Immunol 2015; 135: 123-30.e2.

26. Reddel HK, Busse WW, Pedersen S, et al. Should recommendations about starting inhaled corticosteroid treatment for mild asthma be based on symptom frequency: a post-hoc efficacy analysis of the START study. Lancet 2017; 389: 157-66.

27. Kew KM, Dahri K. Long-acting muscarinic antagonists (LAMA) added to combination long-acting beta 2-agonists and inhaled corticosteroids (LABA/ICS) versus LABA/ICS for adults with asthma. Cochrane Database Syst Rev 2016; 1: CD011721.

28. Lemanske Jr RF, Mauger DT, Sorkness CA, et al. Step-up therapy for children with uncontrolled asthma receiving inhaled corticosteroids. N Engl J Med 2010; 362: 975-85. 
29. Bodzenta-Lukaszyk A, Pulka G, Dymek A, et al. Efficacy and safety of fluticasone and formoterol in a single pressurized metered dose inhaler. Respir Med 2011; 105: 674-82.

30. Papi A, Price D, Sastre J, et al. Efficacy of fluticasone propionate/formoterol fumarate in the treatment of asthma: a pooled analysis. Respir Med 2015; 109: 208-17.

31. Mansur AH, Kaiser K. Long-term safety and efficacy of fluticasone/formoterol combination therapy in asthma. J Aerosol Med Pulm Drug Deliv 2013; 26: 190-9.

32. Papi A, Mansur AH, Pertseva T, et al. Long-term fluticasone propionate/formoterol fumarate combination therapy is associated with a low incidence of severe asthma exacerbations. J Aerosol Med Pulm Drug Deliv 2016; 29: 346-61. 